\title{
O trabalho interdisciplinar no CAPS \\ e a especificidade do trabalho do terapeuta ocupacional*
}

\section{Interdisciplinary work in CAPS and specificity of the work of occupational therapists}

\author{
Angélica Garcia Juns', Selma Lancman²
}

JUNS, A. G.; LANCMAN, S. O trabalho interdisciplinar no CAPS e a especificidade do trabalho do terapeuta ocupacional. Rev. Ter. Ocup. Univ. São Paulo, v. 22, n. 1, p. 27-35, jan./abr. 2011.

\begin{abstract}
RESUMO: Diante das atuais discussões sobre trabalho multiprofissional em saúde e abordagens interdisciplinares, neste trabalho se propõe a reflexão sobre a especificidade da atuação do terapeuta ocupacional nos Centros de Atenção Psicossocial (CAPS). Trata-se de resultados de pesquisa realizada no âmbito do trabalho de conclusão de curso de graduação realizada em 2008, que teve como objetivo compreender a especificidade do trabalho do terapeuta ocupacional no CAPS. A pesquisa se inscreve no campo da investigação qualitativa e o principal método de coleta de dados consistiu em entrevistas semi-estruturadas, analisadas segundo procedimentos metodológicos da análise de conteúdo. Verificou-se que a conformação do serviço exige a presença de profissionais distintos, com competências próprias ao seu campo profissional, ao mesmo tempo que são necessárias competências para a atuação coletiva e um trabalho interdisciplinar que dê conta da atenção ao usuário. Ainda, identifica-se que a caracterização das práticas de trabalho no CAPS não definem a especificidade do terapeuta ocupacional, mas que é possível identificar especificidades na atuação desses profissionais que dizem respeito à abordagem que emprega em suas ações e pelas contribuições que agrega nas discussões com a equipe. Conclui-se que, no desafio para a produção de saúde no contemporâneo, não há necessidade de um campo profissional com fronteiras rígidas e um monopólio sobre determinadas práticas, mas sim de uma perspectiva ampla dos contextos e recursos, em que a especificidade de determinadas profissões agregam contribuições ímpares a esses serviços.
\end{abstract}

DESCRITORES: Terapia ocupacional/tendências; Serviços de saúde mental.

\footnotetext{
* Trabalho de Conclusão de Curso de Graduação realizado no âmbito da pesquisa "Transformação do modelo de atenção pública em saúde mental e seu impacto na saúde mental dos trabalhadores" (Edital promovido pelo Ministério da Saúde em parceria com o Ministério de Ciência e Tecnologia ProcessoCNPq 554503/2005-0). Apresentado oralmente no XI Congresso Brasileiro de Terapia Ocupacional, em Fortaleza, em 16 de outubro de 2009.

1. Terapeuta Ocupacional do Núcleo de Apoio à Saúde da Família pela Associação Congregação de Santa Catarina. E-mail: angelicajuns@ gmail.com

2. Professora Titular do Curso de Terapia Ocupacional do Departamento de Fisioterapia, Fonoaudiologia e Terapia Ocupacional da Faculdade de Medicina da Universidade de São Paulo. E-mail: lancman@usp.br

Endereço para correspondência: LIIST - Departamento de Fisioterapia, Fonoaudiologia e Terapia Ocupacional da FMUSP. Rua Cipotânea, 51. CEP:05360-000.São Paulo,SP.
} 


\section{INTRODUÇÃO}

pesquisa "Transformação do modelo de
atenção pública em saúde mental e seu im-
pacto na saúde mental dos trabalhadores"1 estudou o processo de trabalho de duas equipes de saúde mental diante da transformação do modelo assistencial implementado pela nova política pública de atenção à saúde mental. Nela compreendeu-se que o trabalho nos CAPS produz formas específicas de conceber o cuidado, o que implica na definição de novos perfis profissionais. Perceberam-se determinadas características do processo de trabalho, como as práticas coletivas desenvolvidas, que exigem a constituição de equipes multiprofissionais, e forte cooperação em atividades comuns a diferentes profissionais do serviço (LANCMAN et al., 2008). Este resultado levounos a refletir sobre a relação entre o trabalho no CAPS e as especificidades de cada profissão, e nos motivou a pesquisar as possíveis especificidades da atuação dos terapeutas ocupacionais nos CAPS (JUNS; HA, 2008).

\section{O CAPS, seus profissionais e suas atividades}

A atenção pública à saúde mental no Brasil é atualmente voltada à implantação de projeto político originado pela reforma psiquiátrica, com a desconstrução do modo de gestão centrado no hospital psiquiátrico e construção de um "modelo em rede", ou modelo integral. A aprovação da Portaria Ministerial da Saúde criou em 19/09/2002 a figura orçamentária e organizacional do CAPS e estabeleceu medidas governamentais que permitiram a instauração do atual modelo de atenção à Saúde Mental (BRASIL, 2005).

$\mathrm{O}$ modelo em rede se estrutura com os seguintes serviços do Sistema Único de Saúde (SUS): prontos socorros psiquiátricos; leitos psiquiátricos em hospitais gerais; leitos em hospitais psiquiátricos; serviços residenciais terapêuticos; CAPS tipos I, II e III, infantil (CAPSi) e álcool e drogas (CAPSad); Centros de Convivência (CECCO); Unidades Básicas de Saúde (UBS), com o Programa de Saúde da Família (PSF) e Programa de Agentes Comunitários de Saúde (PACS). O usuário deve ter o CAPS como principal serviço de referência em saúde mental e os demais serviços como suporte na atenção (BRASIL, 2004).

O CAPS prioriza a atenção a portadores de transtornos mentais graves e persistentes e tem suas ações ancoradas na noção do território (a área geográfica e seus habitantes, vizinhança, cenários e instituições). Seus principais objetivos são: prestar atendimento em regime de atenção diária; gerenciar os projetos terapêuticos oferecendo cuidados clínicos eficientes e personalizados; promover a inserção social dos usuários mediante ações intersetoriais que envolvam educação, trabalho, esporte, cultura e lazer, montando estratégias conjuntas de enfrentamento dos problemas; organizar a rede de serviços de saúde mental de seu território; dar suporte e supervisionar a atenção à saúde mental na rede básica através dos PSF e PACS; coordenar, junto com o gestor local, as atividades de supervisão de unidades hospitalares psiquiátricas que atuem no território; e manter atualizada a listagem dos pacientes que utilizam medicamentos psiquiátricos (BRASIL, 2004).

A equipe de um CAPS deve contemplar uma diversidade profissional e cada tipo de CAPS tem características próprias quanto aos tipos e quantidade de trabalhadores. A equipe mínima para estruturação de um CAPS I é: médico psiquiatra ou com formação em saúde mental, enfermeiro, três profissionais de nível superior de categorias diversas (psicólogo, assistente social, terapeuta ocupacional, pedagogo ou outro profissional pertinente) e quatro profissionais de nível médio (técnico e/ou auxiliar de enfermagem, técnico administrativo, educacional e artesão). No CAPS II acrescenta-se mais um profissional de nível superior e dois de nível médio (BRASIL, 2004). Portanto, a contratação de um terapeuta ocupacional é opcional entre os profissionais de nível superior.

De acordo com a Cartilha CAPS (BRASIL, 2004), as atividades a serem realizadas pelos profissionais são: acolhimento, atendimento individual (medicamentoso, psicoterápico, orientação, entre outros), atendimento em grupos (psicoterapia, atividades de suporte social), atendimento em oficinas terapêuticas, visitas domiciliares, atendimento às famílias e atividades comunitárias. Segundo o mesmo documento, o trabalho técnico desenvolvido em um CAPS visa garantir cidadania e emancipação das pessoas com transtornos mentais, ancorados nos referenciais teóricos da reabilitação psicossocial.

\section{A reabilitação psicossocial como estratégia de cuidado e o trabalho no CAPS}

A definição de Reabilitação Psicossocial, dada pela International Association of Psychosocial Rehabilitation

\footnotetext{
1. Edital promovido pelo Ministério da Saúde em parceria com o Ministério de Ciência e Tecnologia (MCT-CNPq/MS-SCTIE-DECIT-CTSaúde) 2005-2008
} 
Services em 1985, é

O processo de facilitar ao indivíduo com limitações, a restauração, no melhor nível possível de autonomia do exercício de suas funções na comunidade. O processo enfatizaria as partes mais sadias e a totalidade de potenciais do indivíduo, mediante uma abordagem compreensiva e um suporte vocacional, residencial, social, recreacional, educacional, ajustados a demandas singulares de cada indivíduo e cada situação de modo personalizado (PITTA, 1996, p.19).

Nessa perspectiva, a reabilitação não é uma tecnologia, mas sim uma abordagem, que abrange os espaços de troca e de tratamento, as diversas situações de vida, definidas por Saraceno (1996) como cenários em que atuamos: habitat (casa); trabalho com valor social (produzir e trocar mercadorias e valores); e rede social (trocar identidades). Compõe-se assim um conjunto de estratégias que visam "aumentar a troca de recursos e de afetos", para que no interior dessa dinâmica se crie um efeito "habilitador" (SARACENO, 1998).

Mângia e Muramoto (2006) explicam que, no caso da pessoa com transtornos mentais, a compreensão sobre a pessoa e sobre a doença deve permear aspectos da desorganização do viver; na perda de vínculos e fragilização das redes de suporte social, na precarização do habitar e na exclusão laboral. O enfrentamento dessas necessidades complexas exige dos profissionais da área aptidão para formular respostas sendo necessário uma redefinição dos processos de trabalho e das equipes. As autoras defendem que os componentes das ações terapêuticas devem estar orientados para uma lógica comum aos trabalhadores, no sentido de evitar a fragmentação dos sujeitos, das necessidades e das ações, produzindo uma atenção integral.

A atuação profissional nesse modelo se inscreve em um conjunto de saberes, instrumentos e práticas articulados, que exige transformações na forma de fazer, pensar o cuidado e no papel das instituições. O cuidado em saúde mental coloca permanentemente em questão as diferentes concepções e estratégias utilizadas pelos profissionais, obrigando-os a reinventarem-se (ROTELLI, 1999 apud MÂNGIA; MURAMOTO, 2006, p.117) Na implantação e desenvolvimento dos CAPS, os trabalhadores vivem um processo de construção de um novo modelo assistencial e são vanguarda de um novo tipo de atendimento.

Essa forma de trabalhar no CAPS constitui um modelo mais horizontal de relação entre os trabalhadores, $\mathrm{o}$ que implica em novos processos de trabalho. Estabelecem-se também novas divisões de tarefas e responsabilidades entre os trabalhadores (RAMMINGER, 2005).

No entanto, o modo de trabalhar não está definido nas normativas que propõem e definem o modelo de atenção e os serviços (como a Portaria 336 de 2002). Sobre esse aspecto, Lancman et al. (2008) apontam para a inexistência de um modo prescrito de trabalhar que dê conta dos possíveis eventos no cuidado nesse novo modelo. A falta de prescrição permite aos trabalhadores uma autonomia na construção do modo de trabalhar, mas deixa-os solitários na resolução de problemas e na busca de soluções, dificultando o reconhecimento das necessidades para o desenvolvimento do trabalho, e consequentemente, a previsão de recursos correspondentes, capacitação, supervisão etc. Assim, os problemas vivenciados, por vezes, transcendem sua formação e até mesmo as condições materiais disponiveis para a realização do trabalho. Para criar esse trabalhador que desenvolverá um trabalho dessa magnitude é necessário que se somem as habilidades desenvolvidas na formação dos vários profissionais que atuam nesse campo. Considerase que a engenhosidade dos diferentes profissionais é que permite que o trabalho seja realizado a contento (LANCMAN et al., 2008).

Sznelwar et al. (2008), a partir de uma análise ergonômica do trabalho (AET) no CAPS, definem a atenção ao usuário do CAPS como fortemente caracterizada pelo trabalho em equipe e reafirmam a importância da utilização de estratégias coletivas para o enfrentamento dos eventos cotidianos. A atuação conjunta durante os atendimentos é apontada na AET como uma necessidade que ultrapassa a visão funcionalista da equipe, sendo também fator de coesão entre os trabalhadores. Os autores ressaltam que a atuação coletiva requer uma construção diária e competências para a cooperação e o desenvolvimento dessas habilidades. Tais competências desenvolvem-se através das experiências acumuladas na vivência no trabalho, nas trocas com a equipe, na elaboração e transformação das práticas e fortalecimento do coletivo.

Segundo Lancman et al. (2008), a equipe se constitui como uma rede de colaboração, importante para a superação das dificuldades, precariedades no trabalho e sofrimentos impostos pelo trabalho com pacientes psiquiátricos graves. Isso requer uma constante recriação da equipe, o que produz "uma sinergia a partir das diferentes competências de cada profissão, da experiência individual e coletiva e do envolvimento dos profissionais" (LANCMAN et al., 2008, p.196). E é nesse contexto que há uma perda do enquadramento dos profissionais no serviço, já que o cuidado e as práticas de saúde são constantemente partilhadas.

Ao mesmo tempo que a conformação do serviço exige a presença de profissionais distintos, com competências próprias ao seu campo profissional, ela exige competências para a atuação coletiva e um trabalho interdisciplinar. 
Considerando que as formações desses profissionais e suas perspectivas para a atenção em saúde mental podem ir em encontro ou em desencontro com o que se propõe com o cuidado oferecido pelo CAPS, o aprendizado da prática da atenção pautada nos preceitos para o desenvolvimento do serviço se contitui ainda como um desafio para eles.

Oliveira e Alessi (2003), em artigo de revisão bibliográfica, discutem o trabalho de enfermeiros em saúde mental e demonstraram que a maioria deles não se sentia preparada para trabalhar na área, a partir dos paradigmas do novo modelo, nem tinham conhecimento das mudanças políticas que vinham ocorrendo. Evidenciaram que os enfermeiros, apesar de reconhecerem os limites do modelo médico-organicista, não se colocavam como agentes transformadores da realidade que denunciavam $\mathrm{e}$ reproduziam o modelo por eles criticado.

Os terapeutas ocupacionais estiveram presentes na atenção a pacientes psiquiátricos graves desde os primórdios da criação da profissão no Brasil. Foram coadjuvantes da luta-antimanicomial e dos novos serviços que decorreram desse movimento, que passou a nortear as atuais políticas públicas de atenção em saúde mental. Participaram igualmente das experiências de criação do CAPS "Prof. Luiz da Rocha Cerqueira", o primeiro CAPS do Brasil, em São Paulo; e da criação de serviços territoriais (Núcleos de Atenção Psicossocial - NAPS), em Santos, durante o final dos anos '80. Essas idéias passaram a fazer parte da formação dos terapeutas ocupacionais, da sua prática assistencial e da sua produção científica, com clara postura a favor da reforma psiquiátrica e do atual modelo de atenção em saúde mental. A particularidade desse histórico profissional é um dos fatores que instiga a investigação sobre a atuação do terapeuta ocupacional no CAPS.

Ainda, entende-se que o terapeuta ocupacional, enquanto participante desse processo de constituição dos serviços, busca em seu cotidiano de trabalho, junto aos demais profissionais, as formas de trabalhar que dêem conta do cuidado em saúde mental. Acredita-se que conhecer as práticas dos terapeutas ocupacionais nos CAPS permitirá também conhecer como essas vivências podem estar modificando as práticas e, por consequência, até mesmo os paradigmas da profissão.

\section{OBJETIVOS}

O objetivo deste artigo é discutir, a partir de uma pesquisa realizada com terapeutas ocupacionais de CAPS na região centro-oeste do município de São Paulo, como esses profissionais compreendem a especificidade do seu trabalho nos CAPS.

\section{ASPECTOS METODOLÓGICOS}

Dentro do contexto da pesquisa relatada (LANCMAN et al., 2008), desenvolveu-se o estudo "A especificidade do trabalho do terapeuta ocupacional no CAPS" (JUNS; HA, 2008), com foco na especifidade da compreensão que os terapeutas ocupacionais tinham a respeito de sua pratica profissional. Utilizou-se abordagem qualitativa, com o uso de entrevistas semi-estruturadas, privilegiando a visão que os trabalhadores têm a respeito do seu trabalho. Participaram do estudo sete terapeutas ocupacionais que trabalhavam, no ano de 2008, nos CAPS I e II da região centro-oeste do município de São Paulo.

Os procedimentos de coleta de dados foram: entrevistas semi-estruturadas, gravadas e transcritas, realizadas no período de julho a setembro de 2008; e o preenchimento de uma grade semanal de atividades no CAPS, para a compreensão das práticas da rotina de trabalho desses profissionais.

As entrevistas ocorreram de acordo com um roteiro que buscou abordar os temas: trajetória profissional prévia, atividades de trabalho e grupos/oficinas coordenadas no CAPS, atuação junto aos outros profissionais, contribuição no conjunto das atividades realizadas, objetivos do trabalho e especificidades do trabalho do terapeuta ocupacional no CAPS.

Os procedimentos para análise dos dados coletados basearam-se em preceitos de Franco (2005) e seguiram as seguintes etapas:

Pré-análise - Leitura minuciosa do material visando selecionar e organizar os conteúdos encontrados,

Exploração do material - busca, no texto, de conteúdos que respondessem aos objetivos especificados,

Tratamento dos resultados - reordenação dos dados obtidos nas etapas anteriores da análise, nas seguintes categorias: a especificidade da TO, diferença na atuação com relação aos outros profissionais, Interpretação dos resultados.

\section{RESULTADOS}

Os resultados estão sistematizados em duas partes. A primeira se refere ao elenco das atividades desempenhadas pelos profissionais pesquisados e a segunda parte apresenta o resultado da análise do conteúdo das entrevistas.

Partindo da leitura das grades em que cada profissional citou suas atividades de trabalho, cabe distinguir que dois terapeutas ocupacionais eram coordenadores dos CAPS estudados, portanto possuíam uma rotina diferenciada, com maior número de atividades técnico-administrativas 
e reuniões sistemáticas em outras instâncias, tais como instituições e serviços pertencentes à rede de saúde e educacional do território.

As atividades de trabalho citadas pelos terapeutas ocupacionais, além das reuniões de equipe, comum a todos, foram: grupos terapêuticos e oficinas; confecção de Jornal Mural; convivência com os usuários; atendimento familiar e individual; Assembléia Geral com os usuários; acolhimento na porta de entrada do serviço; triagem de novos usuários; e atividades sócio-culturais externas ao serviço (passeios, cursos para os usuários, eventos).

Os grupos coordenados pelos terapeutas ocupacionais foram nomeados por: Grupo de Terapia Ocupacional, Terapia Ocupacional e Arte, Atividade Corporal, Taichichuan, Culinária, cuidados pessoais, auto-cuidado, produção (em que são feitas atividades em tear, bijuteria e tapeçaria) e Costura.

A partir da análise das entrevistas, buscou-se identificar as atividades de trabalho que os entrevistados consideram específicas do terapeuta ocupacional e que definem o trabalho que realizam.

Os entrevistados nomearam algumas funções como específicas de alguns profissionais, tais como: prescrever medicamentos (médicos), organização da medicação (farmacêuticos), atendimentos psicoterapêuticos (psicólogos), orientação sobre direitos, deveres e benefícios (assistente social) e entrega de medicação e orientação do seu uso (enfermagem).

No entanto, relatam diversas práticas de trabalho comuns a diferentes profissionais e práticas coletivas por vezes complementares. Relatam que co-participam das atividades, tendo em vista um trabalho de natureza interdisciplinar e mútua responsabilidade por algumas necessidades do serviço.

"A gente faz tudo, né? Marca telefone, faz recepção lá na frente, se precisar faz atendimento familiar. A gente só não receita.(...) acaba fazendo muitas coisas, mas eu acho que cada profissional sabe da especificidade do outro". (entrev. 1)

"Esses trabalhos são complementares, eles se juntam, um está dentro do outro, a gente trabalha em conjunto." (entrev. 2)

Segundo alguns entrevistados, o uso de atividades nas práticas terapêuticas, o trabalho em grupo e a ênfase no fazer, que marcam comumente a especificade dos terapeutas ocupacionais, em espaços como a CAPS são práticas comuns a todos.

"Aqui todo mundo topa muito fazer (...) o que tem tudo a ver com um objeto da Terapia Ocupacional. Eu tenho certeza que poderia ser feito por qualquer outro profissional da equipe. Não ia achar esquisito se outro fizesse, que não fosse a terapeuta ocupacional. Todo mundo faz, a equipe não deixa a gente ser terapeuta ocupacional." (entrev. 3)

Percebe-se que se não há uma especificidade nas atividades desempenhadas por eles, há uma especificidade na forma de trabalhar do terapeuta ocupacional que reside no olhar que caracteriza a sua intervenção.

"Eu acho que tem uma coisa de olhar." (entrev.4)

"Eu não sei se é na atuação, mas nas coisas que a gente é capaz de observar, o que a gente é capaz de extrair daquele momento."(entrev.1)

Verificou-se a utilização das expressões "olhar da Terapia Ocupacional", "forma de ver da Terapia Ocupacional", "uma percepção que quem não é terapeuta ocupacional não tem", "formação do terapeuta ocupacional", entre outras, para explicar uma especificidade na atuação. Identifica-se, assim, através da síntese de diferentes termos utilizados pelos profissionais entrevistados, o olhar do terapeuta ocupacional como sendo para eles a marca da especificidade em seu trabalho no CAPS .

Esse olhar, na fala dos entrevistados, foi relacionado prioritariamente à formação profissional, aos referenciais teóricos que amparam sua atuação e aos saberes acumulados, que constituem a capacidade para compreender e interpretar determinadas situações do trabalho, sobretudo no seu processo de realização, em que, ao fazer, extraem desse processo o material terapêutico da sua intervenção.

"Toda a formação da gente faz com que a gente saiba organizar as atividades, acolher as necessidades e vincular as atividades aos acontecimentos" (entrev.2)

"A minha formação me ajuda a pensar o que vem primeiro.(...)O sujeito da intervenção determina o seu modo e a formação como terapeuta ocupacional me ajuda muito a olhar" (entrev. 5)

Os profissionais relataram que o trabalho no CAPS exige cotidianamente uma aproximação, uma atenção voltada às necessidades individuais e observação de seus comportamentos. Acreditam que os terapeutas ocupacionais desenvolvem uma aproximação diferenciada com o usuário, pela sua formação. 
"Eu acho que trabalhar a escuta do paciente é desenvolvida de uma forma diferenciada na terapia ocupacional." (entrev. 1)

Para eles, a formação em Terapia Ocupacional capacita, para além dessa aproximação, na qualidade da permanência nos espaços de convivência do serviço.

"A Terapia Ocupacional tem muito a acrescentar na convivência (...) facilitar que as pessoas se olhem, se conheçam, conversem. Temos uma facilidade de lidar com essa questão de estar junto." (entrev.2)

"O terapeuta ocupacional tem um perfil de trabalhar convivência e o coletivo, é uma pessoa que mobiliza os pacientes (...)começamos a ver que a convivência era importante e em um ano o CAPS se transformou." (entrev. 7)

Os entrevistados referiram que há noções técnicas e conceituais inerentes à Terapia Ocupacional que permitem atuar com um olhar próprio.

"A Terapia Ocupacional tem um conceito específico de autonomia, diferente dos outros profissionais que tem uma prática muito assistencialista" (entrev. 3)

“essa questão da criatividade, a própria questão da autonomia,(...) a mobilidade do paciente no espaço, a gente trabalha legal isso. Na medida em que a gente tira e guarda coisas, que trabalha a relação do seu corpo com os objetos." (entrev. 1)

As falas dos terapeutas ocupacionais evidenciaram a propriedade de observar e organizar atividades no CAPS e do cotidiano do usuário com um olhar próprio sobre a atividade. Nessa direção, os entrevistados afirmaram que é a especifidade da sua formação que permite olhar para o processo de execução da atividade, decompondo as ações e agindo sobre os usuários em diversas questões envolvidas nesse processo.

"De olhar como o cliente está lidando com o cotidiano dele, com o fazer. Eu acho que a atuação nossa tem um olhar muito especial" (entrev. 4)

"Por mais que as pessoas façam atividade, tem muito uma questão do resultado. a atuação da T.O. é bem diferenciada na questão do percurso da atividade, que esse é um traço que é do terapeuta ocupacional." (entrev. 3)
Outro aspecto expresso é de que os terapeutas ocupacionais evidenciam os contextos externos ao serviço e auxiliam na promoção de atividades fora do CAPS. Sendo que, essas atividades externas são, naquele contexto, entendidas como essenciais para os projetos de reabilitação psicossocial.

"Você transforma aquilo em alguma coisa que reverta no cotidiano deles. Essa visão quem não é terapeuta ocupacional não tem.” (entrev. 6)

Os profissionais referiram que as reuniões de equipe são espaços importantes para a conformação do trabalho e também como lugar de trocas, onde há contribuição desse olhar do terapeuta ocupacional nas discussões de casos e no planejamento do trabalho.

"Nas discussões de caso, são mais claras as diferenças de pontos de vistas e referenciais teóricos, como cada um percebe e interpreta." (entrev. 3)

" temos uma linguagem que acrescenta muito em coisas muito diferentes" (entrev. 1)

"A Terapia Ocupacional amplia a discussão do olhar multidisciplinar." (entrev. 4)

Assim, os terapeutas relataram uma diferença do olhar que contribui com a equipe, dando foco às questões que se relacionam ao cotidiano do paciente e ao fazer humano.

" não trabalhamos só em cima do critério diagnóstico ou da sintomatologia, mas da potencialidade. Por um lado com a questão da patologia e de outro dessa questão do direito à saúde, da potencialidade, do fazer, da práxis. isso é uma especificidade da Terapia Ocupacional." (entrev. 2)

"Aquela preocupação de trocar o sapato, passou desodorante, tomou banho, prendeu o cabelo, limpou o ouvido, não ta com mau hálito, (...). A forma como a gente olha dentro desses espaços contribui para que a equipe possa intervir de outra maneira ou possa ter outro olhar." (entrev. 1)

Por fim, destacaram-se relatos de que, apesar de os meios de fazer o trabalho e "o olhar" sejam diferentes, os objetivos no trabalho são comuns aos diferentes profissionais, diante das demandas da população atendida e do modelo de atenção no CAPS. 
"Não acho que meu objetivo seja diferente da psicóloga, da enfermeira... é um objetivo do CAPS." (entrev. 3)

"O objetivo é comum, é uma discussão que tem há muito tempo, que é a da autonomia, do quanto o sujeito consegue caminhar para fora, para o mundo. O objetivo da equipee não é diferente do objetivo do psicólogo ou do terapeuta ocupacional" (entrev. 4).

Como pudemos observar, a partir dos relatos dos entrevistados, o que caracteriza o trabalho do terapeuta ocupacional no CAPS é o olhar expresso na forma de trabalhar, nas trocas com a equipe e na constituição de práticas voltadas à reabilitação psicossocial. As atividades desenvolvidas muitas vezes são consideradas comuns aos demais profissionais ou compartilhadas pela equipe. As abordagens e percepções do contexto do serviço e dos indivíduos, traduzidos como o olhar desse profissional, é que se destacam como especificidade da atuação do terapeuta ocupacional. Ainda, o trabalho interdisciplinar envolve as práticas no serviço e caracteriza o trabalho no CAPS, o que influencia amplamente para a definição da especificidade profissional.

"CAPSé isso. A gente perde um tanto da especificidade mas tem uma hora que aparece" (entrev.4).

\section{DISCUSSÃO}

Os trabalhadores dos CAPS estão imersos em uma experiência cotidiana que contribui para a construção dos novos perfis profissionais no campo da saúde mental e da reabilitação psicossocial, através de uma abordagem que privilegia as práticas interdisciplinares. Como Sznelwar et al (2008) apontam, o CAPS possui uma prática profissional marcada pelo fortalecimento do coletivo de trabalhadores, onde há trocas de experiências entre a equipe e elaboração conjunta para o desenvolvimento de habilidades coletivas. Os resultados permitem observar que o trabalho interdisciplinar, oriundo da organização do trabalho no serviço, contribui para a percepção dos entrevistados de que no conjunto de práticas de trabalho ocorre a diluição da especificidade devido às características do tipo de trabalho e da instituição onde estão inseridos.

Soares (2007) defende que a terapia ocupacional não é definida somente por seu objeto ou por seus objetivos, pois esses podem ser similares aos de outras profissões, especialmente em equipes profissionais. A autora compreende que as intervenções se diferenciam pelos instrumentos de trabalho e pela maneira de agir do profissional. Assim, entendemos que apesar de alguns métodos de trabalho serem comuns aos diferentes profissionais do CAPS, os terapeutas ocupacionais vêem o seu "olhar" como especifico, o que definiria sua maneira de agir.

Meola (2000) ressalta o aspecto transdisciplinar das intervenções da terapia ocupacional no CAPS e define que esse profissional teria como principal objetivo ampliar o potencial de autonomia dos sujeitos.

Diferentemente das constatações de Oliveira e Alessi (2003), a respeito da dificuldade das enfermeiras de atuarem no novo modelo de atenção em saúde mental, não obtivemos relatos semelhantes entre os terapeutas ocupacionais. Percebe-se uma atuação em consonância com as proposições do novo modelo e isso pode evidenciar que o contexto de formação profissional contribui para as práticas nesse campo.

Benetton (1996), ao discutir a terapia ocupacional na relação com a reabilitação psicossocial, compreende que a postura ativa e arrojada dos profissionais brasileiros no processo de desospitalização psiquiatrica e na produção acadêmica nesse campo contribuíram para o desenvolvimento teórico e conceitual na profissão. A autora concluiu que há uma constituição particular brasileira da profissão, que perpassa a reabilitação tradicional e atinge uma apropriação da reabilitação psicossocial aliada à especificidade profissional.

O compromisso de terapeutas ocupacionais no desenvolvimento de práticas que respondessem às necessidades de seus clientes, ancorados nos preceitos da reabilitação psicossocial, modificaram os paradigmas da profissão e impulsionaram seu desenvolvimento teóricoconceitual (NICÁCIO; MÂNGIA, 2001; MÂNGIA, 2002).

Lima (2006) questiona o que se entende por saude mental, quando tratamos das relações entre Terapia Ocupacional e a Saúde Mental. A autora afirma que é possivel pensar esta relação a partir de dois pontos de vista:

aquele da relação entre a Terapia Ocupacional e o campo da Saúde Mental como um campo específico de atuação e aquele que considera a relação entre Terapia Ocupacional e a produção de saúde mental, tomada em uma perspectiva ampla. Nesta perspectiva ampliada, não estaríamos pensando a saúde mental como uma área de atuação, mas como uma questão que estaria presente na prática dos terapeutas ocupacionais em quaisquer das áreas em que estes venham a atuar (LIMA, 2006, p.119).

Os resultados permitem observar que os terapeutas ocupacionais falam da profissão por vezes como sinônimo de competências específicas para o trabalho em saúde mental. Esse pode ser um elemento essencial para se pensar 
a especificidade da profissão.

Costa e Feriotti (2007) consideram que a construção de uma visão de homem complexa exigiu do terapeuta ocupacional que, em sua prática, buscasse relações entre as diferentes disciplinas, as quais vêm constituindo o campo de conhecimentos da profissão, na construção de um novo caminho que se constrói ao caminhar. A adoção de uma abordagem sistêmica e complexa para a Terapia Ocupacional, considerando a visão de homem, compreensão da relação terapeuta-paciente e a prática terapêutica, na relação entre os diferentes aspectos da saúde humana e do contexto, pressupõe, para as autoras, a construção do conhecimento teórico-prático necessariamente inter e transdisciplinar.

Constata-se então que não só é um aspecto específico da prática no CAPS a adoção de um modelo calcado no trabalho inter e transdisciplinar, que propõe a diluição das especificidades, mas também o desenvolvimento da formação em Terapia Ocupacional; idéia já apontada por Lancman (1998), ao discutir a influência da capacitação dos terapeutas ocupacionais no processo de constituição da profissão no Brasil.

De acordo com Lima (1997), é na tentativa de produzir conhecimento para responder às questões e impasses que nossa prática nos leva a buscar dialogo com diferentes campos de saber. A autora identifica na experiência transdisciplinar a possibilidade de constituição das singularidades e expõe as ideias do filósofo Richard Rorty (1991) de que disciplinas deveriam ser como comunidades, com fronteiras tão fluídas quanto os interesses de seus membros, com o fim do próprio desenvolvimento e compromisso ético com o conjunto do corpo social (RORTY, 1991 apud LIMA, 1997, p.100).

\section{CONCLUSÃO}

A Terapia Ocupacional segue um processo de produção e re-produção de teorias em busca de sustentação de um corpo científico da profissão e ampliação do reconhecimento social e de mercado. Nesse contexto, a constituição da profissão, nos moldes das exigências sociais contemporâneas e no desafio para a produção de saúde não necessita de um campo profissional com fronteiras rígidas e um monopólio sobre determinadas práticas, mas de uma perspectiva ampla, a partir de uma compreensão complexa e dinâmica dos contextos e recursos.

Em busca da manutenção do compromisso ético com a sociedade é que os terapeutas ocupacionais atuam no âmbito das atuais políticas em saúde mental, buscando recursos técnicos e científicos para atender às necessidades da população e para avançar junto ao modelo técnico-assistencial. A prática dos terapeutas ocupacionais nos CAPS pode trazer diversos ganhos à população atendida e às ações em saúde, à medida em que a especificidade da profissão caminha junto às necessidades dos objetos de sua intervenção.

Agradecimentos: Às terapeutas ocupacionais Carolina Maria do Carmo Alonso e Patrícia Su Hyun Ha pela colaboração no processo de pesquisa e parceria para o estudo.

JUNS, A. G.; LANCMAN, S. Interdisciplinary work in CAPS and specificity of the work of occupational therapists. Rev. Ter. Ocup. Univ. São Paulo, v. 22, n. 1, p. 27-35, jan./abr. 2011.

\begin{abstract}
Given the current discussions on the multi and interdisciplinary approaches in health, this work proposes a reflection on the specificity of action of the occupational therapist at the Centers for Psychosocial Care (CAPS). This is the results of the research carried out in the work of conclusion graduate course, in 2008, which aimed to understand the specificity of the work of occupational therapist at CAPS. The research falls within the field of qualitative research and the main method of data collection consisted of semi-structured interviews, analyzed second methodological procedures of content analysis. It was found that the conformation of the service requires the presence of different professional skills with their own professional field, while competencies are needed for collective action and an interdisciplinary work that addresses the user's attention. Still, it is identified that the characterization of working practices in CAPS do not define the specificity of the occupational therapist, but it is possible to identify features on these professionals concerning the approach that employs in its actions and contributions aggregating in discussions with team. It is concluded that the challenge for the production of health in the contemporary, there is no need for a professional field with rigid boundaries and a monopoly on certain practices, but from a broad perspective to an understanding of the contexts and resources, where the specificity of certain professions add unique contributions to these services.
\end{abstract}

KEY WORDS: Occupational therapy/trends; Mental health services. 


\section{REFERÊNCIAS}

BENETTON, M.J. Terapia Ocupacional e reabilitação Psicossocial: uma relação possível? In: Pitta. A, M, (org.). Reabilitação Psicossocial no Brasil. 2a. ed. São Paulo: Hucitec, 1996. pt. IV, cap. 2, p. 143, 1996.

BRASIL. Ministério da Saúde. Secretaria de Atenção à Saúde/ DAPE. Saúde mental no SUS: os centros de atenção psicossocial. Brasília: Ministério da Saúde, 2004.

BRASIL. Ministério da Saúde. Secretaria de Atenção à Saúde/ DAPE. Coordenação Geral de Saúde Mental. Reforma psiquiátrica e política de saúde mental no Brasil. Documento apresentado à Conferência Regional de Reforma dos Serviços de Saúde Mental : 15 anos depois de Caracas. OPAS. Brasília, nov. 2005.

COSTA, C. T.; FERIOTTI, M. L. Terapia ocupacional numa abordagem sistêmica e complexa. In: CAVALCANTI, A.; GALVÃO, C. Terapia ocupacional, fundamentação e prática, Rio de Janeiro: Guanabara Koogan, 2007.

FRANCO. M. L. P. B. Análise de conteúdo. 2a. ed. Brasília: Líber Livros Ed., 2005.

JUNS, A.; HA, P. S. Y. A especificidade do trabalho dos terapeutas ocupacionais no CAPS. São Paulo, 2008. (Trabalho de Conclusão de Curso Terapia Ocupacional) - Faculdade de Medicina da Universidade de São Paulo.

LANCMAN, S.; et al. Politicas públicas e processos de trabalho em saúde mental. Brasília: Paralelo 15, 2008.

LIMA, E. M. F. A. Terapia ocupacional: um território de fronteira? Revista de Terapia Ocupacional da USP, v. 8, n. 2, p. 98-101. 1997.

LIMA, E. M. F. A. A saúde mental nos caminhos da terapia ocupacional. Mundo da Saúde, v. 30, n. 1, p. 117-122, 2006

MÂNGIA, E. F. ; MURAMOTO, M.T. Integralidade e construção de novas profissionalidades no contexto dos serviços substitutivos de saúde mental. Revista de Terapia Ocupacional da USP, v. 17, p. $115-122,2006$.

MÂNGIA, E. F. Contribuições da abordagem canadense na prática de terapia ocupacional centrada no cliente e dos autores da desinstitucionalização italiana para a Terapia Ocupacional em saúde mental. Revista de Terapia Ocupacional da USP, v. 13, n. 3, p. 127-134, 2002.

MÂNGIA, E. F.; NICÁCIO, F. Terapia ocupacional em saúde mental: tendências principais e desafios contemporâneos. In: CARlO, M. M. R. P.; BRATAlOTI, C. C. (Org.) Terapia ocupacional no Brasil: fundamentos e perspectivas. São Paulo: Plexus, 2001

OLIVER, F.C.; NICÁCIO, F. Da instituição asilar ao território: caminhos para produção de sentido nas intervenções em saúde. Revista de Terapia Ocupacional da USP, v. 10, n.2/3, 1999.

OLIVEIRA, A.G.B. \& ALESSI, N.P. O trabalho de enfermagem em saúde mental: contradições e potencialidade atuais. Revista Latinoamericana de Enfermagem, v. 11, n. 3, p. 330-340, 2003.

PITTA, A. M. F. O que é reabilitação psicossocial no Brasil, hoje? In: PITTA, A. M. F. (Org.). Reabilitação psicossocial no Brasil. São Paulo: Hucitec, 1996.

RAMMINGER, T. Trabalhadores de saúde mental: reforma psiquiátrica, saúde do trabalhador e modos de subjetivação nos serviços de saúde mental. 2005. Dissertação (Mestrado - Programa de Pós-graduação em Psicologia Social e Institucional) Universidade Federal do Rio Grande do Sul, Porto Alegre, 2005.

SOARES, L. História da terapia ocupacional. In: CAVALCANTI, A.; GALVÃO, C. Terapia ocupacional, fundamentação e prática. rio de Janeiro: Guanabara Koogan 2007.

SZNELWAR,L; MASCIA,F.; MONTEDO;U.;BRUNORO,C.; ABRAHAO, J. Análise Ergonômica do Trabalho. In: LANCMAN, S.; et al. Políticas públicas e processos de trabalho em saúde mental. Brasília: Paralelo 15, 2008. p. 129-174.

SARACENO, B. A cidadania como forma de tolerância . Tradução: Elisabete Ferreira Mangia. Conferência. Rio de Janeiro, 1998.

SARACENO, B. Reabilitação psicossocial: uma estratégia para a passagem do milênio. In: PITTA, A. M. F. (Org.). Reabilitação psicossocial no Brasil. São Paulo: Hucitec, 1996.

Recebido para publicação: 02/03/11

Aceito para publicação: 08/04/11 